\title{
ORIGINAL
}

\section{LA SALUD BUCODENTAL EN LOS ESCOLARES GALLEGOS. 1995}

Virginia Lorenzo García (1), Ernesto Smyth Chamosa (2), Xurxo Hervada Vidal (1), Rubén Fernández Casal (1), José M. ${ }^{a}$ Alonso Meijide (1), Manuel Amigo Quintana (1), Julia González-Zaera Barreal (1), Agustín Montes Martínez (2) Margarita Taracido Trunk (2) y Teresa Cerdá Mota (1).

(1) Dirección Xeral de Saúde Pública. Santiago de Compostela.

(2) Área de Medicina Preventiva. Facultad de Medicina. Santiago de Compostela.

\section{RESUMEN}

Fundamento: Conocer el estado de la salud bucodental de los escolares gallegos de 6 y 12 años y valorar su aproximación a los objetivos de salud bucodental de la Organización Mundial de la Salud (OMS) para el año 2000.

Métodos: Estudio transversal observacional, realizado en 1995 a un grupo de escolares gallegos de 6 y 12 años elegidos a través de muestreo polietápico por conglomerados. estratificado por provincias y tipo de hábitat.

Resultados: Fueron estudiados 1.288 escolares, 643 en el grupo de 6 años y 645 en el grupo de 12 años. El 46,7\% de los niños de 6 años y el $64,2 \%$ de los de 12 años presentaron caries, lo que supone un índice co de $1.84 \mathrm{y}$ un índice CAO de 1,60 respectivamente.

Conclusiones: Se comprueba una evolución favorable de la salud bucodental de los escolares gallegos. Se han conseguido los objetivos, en salud bucodental, propuestos por la OMS para el año 2000 en la región europea, por lo que se proponen nuevos objetivos.

Palabras clave: Caries dental. Escolares. Prevalencia. Salud escolar. Salud bucodental. Galicia.

\section{ABSTRACT \\ Oral Hygiene among Galician School Children}

Background: To ascertain the condition of the oral hygiene of school children 6 and 12 years of age in Galicia and to assess the degree to which said condition meets the World Health Organization (WHO) oral hygiene objectives for the year 2000 .

Methods: Observational cross-sectional study conducted in 1995 on a group of school children 6 and 12 years of age in Galicia selected by means of a multi-stage sampling by conglomerates, stratified by provinces and type of habitat.

Results: This study was conducted on 1,288 school age children, 643 in the 6-year-old group and 645 in the 12-year-old group. $46.7 \%$ of the 6 year-old children and $64.2 \%$ of the 12 year-olds were found to have cavities, which means an oc index of 1.84 and a $A O C$ index of 1.60 , respectively.

Conclusions: A trend toward improvement in the oral hygiene of school-age children in Galicia has been found. The oral hygiene-related objectives recommended by the WHO for the year 2000 in the region of Europe have been acconplished, as a result of which further objectives are recommended.

Key words: Prevalence. School Health. Oral hygiene. Galicia. Dental decay. School children

\section{INTRODUCCIÓN}

La salud bucodental, en particular la caries, despierta gran interés entre los profesionales

Correspondencia:

Virginia Lorenzo García.

Servicio de Actividades Preventivas.

Dirección Xeral de Saúde Pública.

Avenida Camiño Francés 10 bajo.

15771. Santiago de Compostela. A Coruña. y autoridades sanitarias, por ser la patología más prevalente en la sociedad desarrollada, especialmente entre los niños en edad escolar'1.

En España, el nivel de prevalencia de caries entre la población infantil muestra una tendencia decreciente ${ }^{2-6}$, aunque permanece como un problema de salud importante en la Comunidad Autónoma de Galicia, recogido en el Plan de Salud 1993-97 de la Consellería de Sanidad y Servicios Sociales? 
La OMS en el Programa «Salud para todos en el año 2000», establece como objetivos de salud oral para la región europea, una prevalencia de caries en niños de 6 años no superior al $50 \%$ y un índice $\mathrm{CAO}$ a los 12 años menor o igual a $3^{8,9}$.

En la Comunidad Autónoma Gallega, desde el trabajo de Taracido ${ }^{10}$ en el año 1990,que encontró una prevalencia de caries del $87,9 \%$ en escolares de 6 a 14 años, no se disponía de datos de la salud bucodental entre la población infantil y como la OMS recomienda actualizar cada 5 años estos datos ${ }^{9}$, se realizó un estudio epidemiológico en 1995 con el objetivo de conocer el estado de la salud bucodental de los escolares de 6 y 12 años de nuestra comunidad y valorar su aproximación a los objetivos de la OMS de salud para todos en el año 2000 para la región europea.

\section{MATERIAL Y MÉTODOS}

Se realizó un estudio transversal, observacional, durante 1995 en los escolares gallegos. Se estimó que la población total objetivo del estudio era de 34.000 escolares de 6 años y 24.500 de 12 años, siguiendo los datos publicados por el Instituto Gallego de Estadística $^{11}$. Entre ellos se obtuvo una muestra representativa, a través de un sistema de muestreo por conglomerados bietápico, estratificado por provincias y hábitat (poblaciones de más de 50.000 habitantes, de 10.000 a 50.000 y menores de 10.000 habitantes). En una primera etapa, en cada estrato se seleccionaron los conglomerados mediante muestreo aleatorio proporcional a su tamaño (42 colegios seleccionados).

El tamaño muestral se calculó teniendo en cuenta estimaciones de la prevalencia de caries en cada estrato. Se fijó un error absoluto de muestreo del $3 \%$, con un nivel de confianza del $95 \%$ y una estimación de ausencias del $10 \%$. El tamaño resultante fue de 700 escolares para cada grupo. Cada conglomerado resultó estar formado por $17 \mathrm{ni}$ - ños por cada escuela y grupo de edad. En cada colegio se seleccionaron los escolares mediante muestreo aleatorio sistemático.

Todos los niños seleccionados fueron sometidos a una exploración bucodental llevada a cabo por 4 equipos formados por un odontólogo y un higienista, previamente formados y unificados con un alto grado de concordancia interobservador $(83 \%-95 \%)$ para todos los posibles estados de la pieza dental ${ }^{12,13}$. A lo largo del estudio se evaluó la concordancia intraobservador repitiendo una exploración de cada diez, sin que el explorador tuviera conocimiento de ello.

Se han estudiado las siguientes variables: oclusión dental siguiendo los criterios de la $\mathrm{OMS}^{12}$ y su clase siguiendo la clasificación de Angle ${ }^{14}$, la presencia de placa (índice de Silnes y Loes) y el estado periodontal $(\mathrm{CPITN})^{12}$. Para el estudio de la higiene y del estado periodontal se dividió la boca en sextantes, obteniéndose la valoración de cada niño a partir del peor estado de los sextantes estudiados; también se calculó la proporción de sextantes afectados del total de los explorados expresada en tanto por ciento.

Todas las piezas dentales fueron exploradas con espejo y sonda ${ }^{12}$. Se evaluaron las necesidades de tratamiento, se elaboraron los índices CAO y co según se considerasen las piezas definitivas o deciduales y se evaluó el estado del molar de los 6 años.

Todo el trabajo de campo se realizó en el primer semestre de 1995 y fueron solicitados los permisos necesarios de padres y profesores para poder llevar a cabo la exploración de los niños seleccionados. La exploración se realizó en los gabinetes de rcconocimicnto médico de las escuelas con los escolares tumbados en camilla e iluminación artificial.

El análisis estadístico se llevó a cabo con el programa SPSS versión 6.1.3 para windows. Para estimar los intervalos de confianza de la prevalencia de caries se tuvo en cuenta el diseño complejo de la muestra. 


\section{RESULTADOS}

El número final de escolares estudiados fue de 643 de 6 años y 645 de 12 años.

\section{Escolares de 6 años}

\section{Oclusión dental}

De los 643 escolares revisados el 70,5\% no presentan anomalías, en el $13,1 \%$ son leves y en el $16,5 \%$ severas. El $76.7 \%$ tenían oclusión clase I, el $21,9 \%$ clase II y el $1,4 \%$ clase III en el lado derecho y clase $I$ en el $79,3 \%$, clase II en el $19,7 \%$ y clase III en el $1 \%$ en el lado izquierdo. El resalte es de más de $8 \mathrm{~mm}$ en el $3,3 \%$ y negativo en el $1,17 \%$. La sobremordida es de menos de $3 \mathrm{~mm}$ en el $82,02 \%$, en el $10,7 \%$ de más, en el $2,63 \%$ es oclusión borde a borde y en el $4,65 \%$ tienen mordida abierta. La línea media estaba desviada a la derecha en el $1,7 \%$ de los casos y a la izquierda en el $1,4 \%$.

\section{Estado periodontal}

Teniendo en cuenta el peor estado en cada sextante, se observa que el $44,9 \%$ de los niños tienen todos los sextantes sanos, en el $42,9 \%$ el peor estado del sextante era el sangrado y en el $12,1 \%$ de los niños era el cálculo. Debido al estado de intercambio de las piezas dentales en este grupo no se ha podido estudiar el 16,5\% de los sextantes. El 68,1\% de todos los sextantes están sanos, el 11,2\% presenta sangrado a la exploración y el 3,7\% cálculo. La mayor proporción de sextantes con cálculo se localiza en el sextante central inferior $(7,9 \%)$, y el mayor porcentaje de sextantes que sangran a la exploración en los sectores posteriores superiores e inferiores.

\section{Higiene dental}

En el grupo de 6 años, 49 niños $(7,6 \%)$ tienen todos los sextantes sin placa. El peor estado de higiene dental entre los niños es placa en el $25,7 \%$ de los explorados, placa en el tercio gingival en el $42,1 \%$ y abundante placa en el 23,6\% de los escolares. De la totalidad de los sextantes estudiados, el $32,1 \%$ están limpios, el 28,9\% tienen placa en el borde gingival, $31,9 \%$ en $1 / 3$ del diente y en el $6,7 \%$ de los casos sobrepasa 1/3 del diente. La mayor proporción de sectores limpios corresponde a los sextantes anteriores superior e inferior con $52,9 \%$ y 48,45 respectivamente. La presencia de placa en el borde gingival es mayor en los sectores posteriores inferiores. Las zonas de mayor localización de placa son los sextantes posteriores superiores e inferiores.

\section{Caries}

La prevalencia de caries a los 6 años y los índices CAO y co en esta edad se recogen en las tablas 1, 2 y 3 respectivamente. Considerando la intensidad de la afectación, se observó que el 29,2\% de los niños de 6 años tenían afectadas entre 1 y 4 piezas tempora-

Tabla 1

Prevalencia de caries entre los escolares de 6 y 12 años

\begin{tabular}{|c|c|c|c|c|c|c|}
\hline \multirow[b]{2}{*}{ Tipo dentición } & \multicolumn{3}{|c|}{ Escolares de 6 años } & \multicolumn{3}{|c|}{ Escolares de 12 años } \\
\hline & $N .^{o}$ & $\%$ & $\begin{array}{c}\text { Intervalo de } \\
\text { confianza } \\
(95 \%)\end{array}$ & $N .^{\circ}$ & $\%$ & $\begin{array}{c}\text { Intervalo de } \\
\text { confianza } \\
(95 \%)\end{array}$ \\
\hline Ambas denticiones & 300 & 46,7 & $(41,3,52,1)$ & 414 & 64,2 & $(59,0,69,4)$ \\
\hline Dentición definitiva & 35 & 5,4 & $(3,0,7,9)$ & 375 & 58,1 & $(52,8,63,5)$ \\
\hline Dentición temporal & 294 & 45,7 & $(40,3,51,1)$ & 91 & 14,1 & $(10,4,17,9)$ \\
\hline Molar 6 años & 30 & 4,7 & $(2,4,6,9)$ & 359 & 55,7 & $(50,3,61,0)$ \\
\hline
\end{tabular}


les y el $16,5 \%$ tenían 5 o más piezas alteradas. El 54,3\% de los alumnos no presentaban alteraciones en la dentición temporal. En este grupo etario, la afectación en las piezas definitivas se limitó a que el $5,4 \%$ de los niños tenían afectadas entre 1 y 4 piezas. El 94,6\% restante de los alumnos no presentaron alteraciones en su dentición definitiva.

Tabla 2

Piezas definitivas afectadas en los escolares de 6 y 12 años. Índice CAO a los 6 y 12 años

\begin{tabular}{|c|c|c|c|c|c|}
\hline \multicolumn{6}{|c|}{ Escolares de 6 años } \\
\hline & N..$^{\circ}$ Medio & $\begin{array}{c}\text { Error } \\
\text { Estándar }\end{array}$ & Minimo & Máximo & $\begin{array}{l}\text { Piezas } \\
\text { revisadas }\end{array}$ \\
\hline CARIADAS & 0,07 & 0,01 & 0 & 3 & 42 \\
\hline OBTURADAS & 0,01 & 0,00 & 0 & 2 & 8 \\
\hline AUSENTES & 0,00 & 0,00 & 0 & 1 & 1 \\
\hline $\mathrm{CAO}$ & $0,08^{*}$ & 0,02 & 0 & 4 & 51 \\
\hline \multicolumn{6}{|c|}{ Escolares de 12 años } \\
\hline & N. ${ }^{\circ}$ Medio & $\begin{array}{l}\text { Error } \\
\text { Estándar }\end{array}$ & Mínimo & Máximo & $\begin{array}{l}\text { Piezas } \\
\text { revisadas }\end{array}$ \\
\hline CARIADAS & 0,77 & 0,08 & 0 & 11 & 495 \\
\hline OBTURADAS & 0,76 & 0,07 & 0 & 11 & 492 \\
\hline AUSENTES & 0,07 & 0,01 & 0 & 4 & 45 \\
\hline $\mathrm{CAO}$ & $1,60^{* *}$ & 0,11 & 0 & 15 & 1032 \\
\hline
\end{tabular}

* Intervalo de confianza $(95 \%):(0,03,0,12)$.

** Intervalo de confianza (95\%): $(1,38,1,81)$.

Tabla 3

Piezas temporales afectadas en los escolares de 6 y 12 años. Índice co a los 6 y 12 años

\begin{tabular}{|c|c|c|c|c|c|}
\hline \multicolumn{6}{|c|}{ Escolares de 6 años } \\
\hline & $N^{\circ}$ Medio & $\begin{array}{c}\text { Error } \\
\text { Estándar }\end{array}$ & Minimo & Máximo & $\begin{array}{l}\text { Piezas } \\
\text { revisadas }\end{array}$ \\
\hline CARIADAS & 1,62 & 0,15 & 0 & 15 & 1041 \\
\hline OBTURADAS & 0,22 & 0,06 & 0 & 8 & 144 \\
\hline $\mathrm{CO}$ & $1,84^{*}$ & 0,16 & 0 & 15 & 1185 \\
\hline \multicolumn{6}{|c|}{ Escolares de 12 años } \\
\hline & $N^{\circ}$ Medio & $\begin{array}{c}\text { Error } \\
\text { Estándar }\end{array}$ & Mínimo & Máximo & $\begin{array}{c}\text { Piezas } \\
\text { revisadas }\end{array}$ \\
\hline CARIADAS & 0,24 & 0,04 & 0 & 6 & 152 \\
\hline OBTURADAS & 0,04 & 0.01 & 0 & 3 & 25 \\
\hline $\mathrm{CO}$ & $0,27 * *$ & 0,05 & 0 & 6 & 177 \\
\hline
\end{tabular}

* Intervalo de confianza (95\%): $(1.53,2,15)$.

** Intervalo de confianza (95\%): $(0,18,0,36)$. 
Tabla 4

Escolares de 6 años según el tipo de caries en cada dentición

\begin{tabular}{|c|c|c|c|c|c|c|c|c|}
\hline \multirow{2}{*}{ Dentición } & \multicolumn{2}{|c|}{ Sin caries } & \multicolumn{2}{|c|}{$\begin{array}{c}\text { Con } \\
\text { caries tratadas }\end{array}$} & \multicolumn{2}{|c|}{$\begin{array}{c}\text { Con caries } \\
\text { parcialmente tratadas }\end{array}$} & \multicolumn{2}{|c|}{$\begin{array}{l}\text { Con caries } \\
\text { sin tratar }\end{array}$} \\
\hline & $N^{\circ}$ & $\%$ & $N .^{\circ}$ & $\%$ & $N .^{o}$ & $\%$ & $N^{o}$ & $\%$ \\
\hline Ambas & 343 & 53,3 & 19 & 3 & 40 & 6,2 & 241 & 37,5 \\
\hline Definitiva & 608 & 94,6 & 6 & 0,9 & 2 & 0,3 & 27 & 4,2 \\
\hline Temporal & 349 & 54,3 & 18 & 2,8 & 38 & 5,9 & 238 & 37 \\
\hline
\end{tabular}

Las necesidades de tratamiento en este grupo quedan reflejadas en la tabla 4.

\section{Escolares de 12 años}

\section{Oclusión dental}

De los 645 escolares revisados el $46,8 \%$ no presentan anomalías, en el $29,3 \%$ son leves y en el $23,9 \%$ severas. El $73,8 \%$ tienen oclusión clase I, el $21,3 \%$ clase II y el $4,7 \%$ clase III en el lado derecho y clase I en el $75,6 \%$, clase II en el $20,8 \%$ y clase III en el $3,5 \%$ en el lado izquierdo. El resalte es de más de $8 \mathrm{~mm}$ en el $6,6 \%$ y negativo en el $0,9 \%$. La sobremordida es de menos de $3 \mathrm{~mm}$ en el $54,3 \%$, en el $41,6 \%$ de más, en el $2,3 \%$ es oclusión borde a borde y en el $1,7 \%$ tienen mordida abierta. La línea media estaba desviada a la derecha en el $1,9 \%$ de los casos y a la izquierda en el $4,8 \%$.

\section{Estado periodontal}

Teniendo en cuenta el peor estado en cada sextante, se observa que el $33,39 \%$ de los niños tienen todos los sextantes sanos, en el $23,7 \%$ el peor estado del sextante era el sangrado y en el $42,9 \%$ de los niños era el cálculo. El $60 \%$ de todos los sextantes están sanos, el $24,7 \%$ presenta sangrado a la exploración, el 14,6\% cálculo y se excluyeron el $0,8 \%$ por no poder ser explorados adecuadamente. La localización de los sectores sanos es mayor en la arcada superior y en el sector central, siendo la inversa en la arcada inferior. La mayor proporción de sectantes que sangran a la exploración son los posteriores inferiores y la mayor proporción de sectores con sarro se da en el sextante anterior e inferior y en los posteriores superiores.

\section{Higiene dental}

En el grupo de 12 años, 56 niños $(8,7 \%)$ tienen todos los sextantes sin placa. El peor estado de higiene dental entre los niños es placa en el $25,3 \%$ de los explorados, placa en el tercio gingival en el $45 \%$ y abundante placa en el $21 \%$ de los escolares. El 28,5\% de los sectores estaban limpios, el $30,5 \%$ tenían placa en el borde gingival, el $33,6 \%$ en $1 / 3$ del diente y el $6,7 \%$ de los sectores en más de 1/3. Los sectores anteriores son los más limpios, $51,2 \%$ el superior y $42,3 \%$ el inferior siendo los sectores superiores y posteriores los que presentan más placa.

\section{Caries}

La prevalencia de caries a los 12 años y los índices CAO y co en esta edad se recogen en las tablas 1, 2 y 3 respectivamente. Considerando la intensidad de la afectación, se observó que el 13,3\% de los niños de 12 años tenían afectadas entre 1 y 4 piezas temporales y el $0,8 \%$ tenían 5 o más piezas alteradas. El $85,9 \%$ de los alumnos no presentaban alteraciones en la dentición temporal. En las piezas definitivas se manifestó la afectación de 1 a 4 piezas en el $51,2 \%$ de los niños y de 5 o más piezas en 
el $7 \%$ de los alumnos. El 41,8\% restante de los alumnos no presentaron alteraciones en su dentición definitiva.
Las necesidades de tratamiento de los niños de este grupo quedan reflejadas en la tabla 5 .

Tabla 5

Escolares de 12 años según el tipo de caries en cada dentición

\begin{tabular}{|c|c|c|c|c|c|c|c|c|}
\hline \multirow{2}{*}{ Dentición } & \multicolumn{2}{|c|}{ Sin caries } & \multicolumn{2}{|c|}{$\begin{array}{c}\text { Con } \\
\text { caries tratadas }\end{array}$} & \multicolumn{2}{|c|}{$\begin{array}{c}\text { Con caries } \\
\text { parcialmente tratadas }\end{array}$} & \multicolumn{2}{|c|}{$\begin{array}{l}\text { Con caries } \\
\text { sin tratar }\end{array}$} \\
\hline & $N .^{\circ}$ & $\%$ & $N .^{o}$ & $\%$ & $N .^{\circ}$ & $\%$ & $N^{\circ}$ & $\%$ \\
\hline Ambas & 231 & 35,8 & 139 & 21,6 & 89 & 13,8 & 186 & 28,8 \\
\hline Definitiva & 270 & 41,9 & 151 & 23,4 & 69 & 10,7 & 155 & 24,0 \\
\hline Temporal & 554 & 85,9 & 11 & 1,7 & 5 & 0,8 & 75 & 11,6 \\
\hline
\end{tabular}

\section{DISCUSIÓN}

Los primeros datos sobre la salud oral en Galicia son del año 1969, aportados por Domínguez Carmona y colaboradores ${ }^{15}$, que observaron una prevalencia del $83,1 \%$ y 3,09 piezas cariadas de media en la población escolar del ayuntamiento de Santiago de Compostela. Gimeno de Sande, el mismo año, observó una prevalencia del 79,3\% en los niños de la provinciá de Orense con una media de 3,5 piezas cariadas por niño ${ }^{2}$. Aunque la metodología no es comparable, la prevalencia de caries en nuestro estudio con valores del $46,7 \%$ y del $64,2 \%$ y unos índices co de 1,82 y CAO de 1,60 para los 6 y 12 años respectivamente, se alejan de los valores referidos por los autores anteriores $^{2,15}$ y por Taracido ${ }^{10}$ en el año 90.

Cuenca $^{6}$ recoge los índices $\mathrm{CAO}$ de diversos estudios realizados a lo largo del tiempo a niños de 12 años en diferentes comunidades autónomas y en España. En esta revisión ${ }^{6}$ se aprecia que el valor más próximo al de los escolares gallegos es ei CAO 1,34 en los escolares de la ciudad de Barcelona.

El índice co a los 6 años $(1,84)$ es bajo entre los escolares gallegos si se compara con los valores encontrados en diferentes estudios de la literatura realizados entre los años 1969 y 1993 y recogidos por Romero
Ruiz y colaboradores ${ }^{16}$. Su valor es más cercano al de la comunidad de Murcia $(1,34)$ y se aleja del valor del País $\operatorname{Vasco}^{3}(2,59)$.

E1 molar de los 6 años, habitualmente, es la primera pieza permanente que hace su erupción en la cavidad bucal y la que más se ve afectada por el proceso cariogénico, de ahí la importancia de su estudio. A los 6 años el $4,7 \%$ de los escolares gallcgos y el $55,7 \%$ a los 12 años presentaron caries en esta pieza dental. En la comunidad de Murcia ${ }^{5}$ los valores son de $11,35 \%$ a los 6 años y $51,95 \%$ a $\operatorname{los} 12$ años.

Otros autores ${ }^{17-19}$ estudiando población infantil pero abarcando otros grupos de edad, encuentran diferentes valores en la prevalencia de caries y en los índices co y CAO. En Europa varían entre los diversos países manteniéndose altos en la zona centro y este del continente $^{20}$.

El porcentaje de sextantes que presentan enfermedad periodontal se duplica en el grupo de 12 años respecto al de 6 , lo que confirma que esta patología afecta más a los segmentos de población de mayor edad.

Es necesario un mayor nivel de higiene bucodental, ya que solo el 7,6\% de los escolares de 6 años y el 8,7\% de 12 tenían todos los sectores sin placa. En estudios realizados en otras comunidades se constató una impor- 
tante mejora de los indicadores de afectación y un aumento en la atención den$\operatorname{tal}^{18,19,21}$.

A la vista de los resultados del estudio se puede afirmar que en Galicia se han conseguido los objetivos propuestos por la OMS para el año 2000 en la Región europea, ya que la prevalencia de caries de $46,7 \%$ en niños de 6 años y el índice CAO a los 12 años de 1,87 , son inferiores a los valores recomendados ${ }^{8,9}$. Por ello, se proponen como objetivos a alcanzar en nuestra comunidad en el año 2000, un índice CAO a los 12 años inferior a 1,5 y una prevalencia de caries menor de $45 \%$ en los niños de 6 años.

La mejora en los indicadores de salud bucodental alcanzada por los escolares gallegos puede ser atribuida a causas muy diversas: la mejora del nivel de vida, el aumento del número de profesionales de la salud bucodental y su integración en los centros de atención primaria, la mayor conciencia de padres y maestros en la importancia de la educación sanitaria, o el impacto del Programa Gallego de Salud Bucodental en la escuela, puesto en marcha en el curso escolar 86-87 desde la Dirección General de Salud Pública de la Consellería de Sanidad y Servicios Sociales con el objetivo de mejorar la salud bucodental y prevenir la caries mediante la promoción de educación para la salud bucodental ${ }^{22}$ y un sistema de colutorios fluorados semanales ${ }^{23}$.

A pesar de estos datos favorables, no se debe restar importancia a las enfermedades de la cavidad oral ya que sigue siendo la patología más prevalente y la eficacia de las medidas preventivas correctamente aplicadas está altamente demostrada en este problema de salud.

\section{BIBLIOGRAFÍA}

1. I. Dolado, P. Casañas, M. Nebot y C. Manau. Prevalencia de caries y factores asociados en escolares de 12 años de Barcelona. Aten Primaria 1996; 18 (3): $111-115$
2. Gimeno de Sande A.; Sánchez Fernández B.; Viñes Rueda JJ; Gómez Pomar F.; Mariño Aguiar F. Estudio epidemiológico de la caries dental y la patología bucal en España. Rev San Hig Publica 1971. 45: $362-341$.

3. Departamento de Sanidad y Consumo del Gobierno Vasco: Estudio epidemiológico de la salud bucodental infantil en la comunidad autónoma vasca. Vitoria: Servicio de publicaciones, Gobierno Vasco; 1990 .

4. Estudio epidemiológico sobre la salud bucodental en escolares asturianos. Vitoria: Consejería de Sanidad y Servicios Sociales; 1994.

5. Consejería de sanidad. Encuesta de salud bucodental en escolares de la región de Murcia. Murcia: Consejería de sanidad; 1990.

6. Cuenca E, Álvarez M T. Evolución de la salud hucodental en Fspaña, en los últimos 20 años. Archiv Odontoestomatol Prev Com 1991; 3 (1): 33-39.

7. Consellería de Sanidade e Servicios Sociais: Plan de Saúde de Galicia 1993-1997. Santiago de Compostela: Consellería de Sanidade e Servicios Sociais; 1993.

8. OMS. Seric de informes técnicos. Gincbra; 1989. Informe técnico n. ${ }^{\circ} 782$ : 1-77.

9. OMS. Planificación de servicios de salud bucodental. Ginebra; 1981. Publicación n. 53: 1-52.

10. Taracido Trunk, M: Estudio epidemiológico de la caries dental en la población escolar de Galicia. [Tesis doctoral]. Santiago de Compostela: Universidad de Santiago de Compostela; 1990.

11. Instituto Galego de Estadística. Movemento Natural da Poboación-Nacementos Galicia anos 1991 e 1992. Xunta de Galicia. Santiago de Compostela: Consellería de Economía e Facenda; 1993.

12. Word health Organization. Oral Health Surveys. Basic methods 3. ${ }^{a}$ ED. Geneva: Word health Organization; 1987.

13. Rubio Colavida JM, Robledo de Dios T, Llodra Calvo JC, Simón Salazar F, Joaquín Artacoz Osés, Vicente L. González Andrés y José María GarcíaCamba de la Muelia. Criterios mínimos de los estudios epidemiológicos de salud dental en escolares. Rev Esp Salud Pública 1997; 71: 231-242.

14. Canut J.A. Ortodoncia Clínica. Barcelona: Mason; 1992

15. Domínguez Carmona M, Rodríguez Míguez, Facel H, Gestal JJ. y cols. Estudio epidemiológico de la caries dental en los escolares de Santiago. Rev San Hig Pública 1970. 44: 15-38. 
16. Romero Ruiz M M, Serrano González A, Álvarez-Ossorio García de Soria M R y Zafra Mezcua J A. Prevalencia de la caries dental en la población de 6 años de San Fernando (Cádiz). Aten Primaria. 1996; 18 (8): 431-435.

17. A. Sicilia, J. Cobo, B. Noguerol, R. Hernández, V. Lucas, J. Ainamo, A. Bascones y J. S. López Arranz. Prevalencia de caries en los niños y jóvenes escolares españoles de siete, doce y quince a diecinueve años. Av Odontoestomat 1990; 6 (6): 323-330.

18. M. E. Calle Purón, A. Gil Miguel, M. L. Lasheras Lozano, J. Rey Calero, V. Domínguez Rojas. Estudio epidemiológico y evolución de la caries dental en colegios de Madrid. Avan Odontoestomatol 1990; 6 (10): 611-614.

19. M C. Ayerbe Mateo-Sagasta, M. Caballero García, A. Jara Siguero y F. J. Sanz Serrulla

20. Estudio de la caries dental en escolares de una zona urbana de la Comunidad de Madrid. Medifam. 1997; 7 (4): 226-232.
21. ORCA Saturday Afternoon Symposium 1995. The Prevalence of Dental Caries in Europe 1990-1995. Caries Res. 1996: 30: 237-255.

22. González de Dios J, Monerris García E, Ortega Pastor E, Quesada de la Gala C, Gómez Gómez R, Calpena Vera M T, Ruiz Amorós E, Moya Benavent $\mathrm{M}$. Estudio de hábitos de higiene bucodental en preadolescentes y adolescentes de dos colegios urbanos y dos rurales. An Esp Pediatría 1995. 45 (1): $14-20$.

23. Guía «A experiencia de educar para a saúde na escola». Educación bucodental. Programa de Educación para a Saúde na Escola. Santiago de Compostela: Consellería de Sanidade e Servicios Sociais y Consellería de Educación e Ordenación Universitaria; 1992.

24. E. Smyth, M. Taracido, JJ Gestal. El flúor en la prevención de la caries dental. Santiago de Compostela: Consellería de Sanidade e Servicios Sociais. Santiago; 1991. 